\title{
Multigap Superconductivity in the Ferromagnetic Superconductor UCoGe Revealed by Thermal Conductivity Measurements
}

\author{
Ludovic Howald, ${ }^{1,2}$ Mathieu Taupin,, ${ }^{3,4}$ and Dai Aoki ${ }^{3,4,5}$ \\ ${ }^{1}$ Physik-Institut der Universität Zürich, Winterthurerstrasse 190, 8057 Zürich, Switzerland \\ ${ }^{2}$ Swiss Light Source, Paul Scherrer Institut, 5232 Villigen, Switzerland \\ ${ }^{3}$ Institut Nanosciences et Cryogénie, Commissariat à l'Énergie Atomique et aux Énergies Alternatives, 38054 Grenoble, France \\ ${ }^{4}$ University Joseph Fourier, 38041 Grenoble, France \\ ${ }^{5}$ Institute for Materials Research, Tohoku University, Oarai, Ibaraki 311-1313, Japan
}

Correspondence should be addressed to Ludovic Howald; ludovic.howald@psi.ch

Received 7 July 2014; Revised 15 September 2014; Accepted 17 September 2014; Published 9 October 2014

Academic Editor: Zhiqiang Mao

Copyright (C) 2014 Ludovic Howald et al. This is an open access article distributed under the Creative Commons Attribution License, which permits unrestricted use, distribution, and reproduction in any medium, provided the original work is properly cited.

We performed thermal conductivity measurements on a single crystal of the ferromagnetic superconductor UCoGe under magnetic field. Two different temperature dependencies of the thermal conductivity are observed, for $\vec{H} \| \vec{b}$ : linear at low magnetic field and quadratic for magnetic field larger than 1 Tesla. At the same field value, a plateau appears in the field dependency of the residual term of thermal conductivity. Such observations suggest a multigap superconductivity with a line of nodes in the superconducting gap.

\section{Introduction}

The orthorhombic heavy fermion system UCoGe, discovered in 2007 [1], is one of the few compounds exhibiting long range coexistence between weak itinerant ferromagnetism (magnetic moment $m_{0} \cong 0.07 \mu_{B}[2]$ ) and superconductivity. Such coexistence is attested by the observation of two bulk phase transitions in specific heat measurements [1]. $\mu \mathrm{SR}$ and NQR measurements on different samples $[3,4]$ reveal that the compound is fully ferromagnetic below the Curie temperature $\left(T_{\text {Curie }} \cong 2.4 \mathrm{~K}\right.$ ) while about $50 \%$ of the sample is superconducting below $T_{\mathrm{SC}} \cong 0.5 \mathrm{~K}[4,5]$.

The upper critical field is extremely anisotropic, exceeding 16 Tesla for $\vec{H} \| \vec{a}$ and $\vec{H} \| \vec{b}$ while it reaches only 0.5 Tesla for $\vec{H} \| \vec{c}$ [6], the easy magnetization axis [2]. The coexistence of ferromagnetism and superconductivity and the observed extremely high upper critical field suggest the realization of unconventional superconductivity with equal spin pairing (triplet) [7]. Such a superconductor is inherently two-band (one for the up and one for the down spins); however it is not known whether the two bands are superconducting or only one, in analogy to the $\mathrm{Al}$ phase of ${ }^{3} \mathrm{He}$, and whether the decoupling between the bands is large enough to induce multigap superconductivity.

Multigap superconductivity is quite common. First observed in Nb-doped $\mathrm{SrTiO}_{3}$ [8], it is found in $\mathrm{MgB}_{2}$ [9], various heavy fermions $[10,11]$, cuprates [12], and pnictides [13] systems. Here we report evidences for multigap superconductivity in the ferromagnetic superconductor UCoGe.

For a two-band ferromagnetic superconductor, due to the crystal structure and the strong spin orbit coupling [14], only two types of odd parity superconducting states are possible [15]. These states differ by the position of their nodes, lying either on the northern and southern poles of the Fermi surface $k_{x}=k_{y}=0$ or on the line of equator $k_{z}=0$. The symmetry of the superconducting gap has a small effect on the shape of the upper critical field, which was used by Hardy and Huxley [16] to suggest a superconducting state 


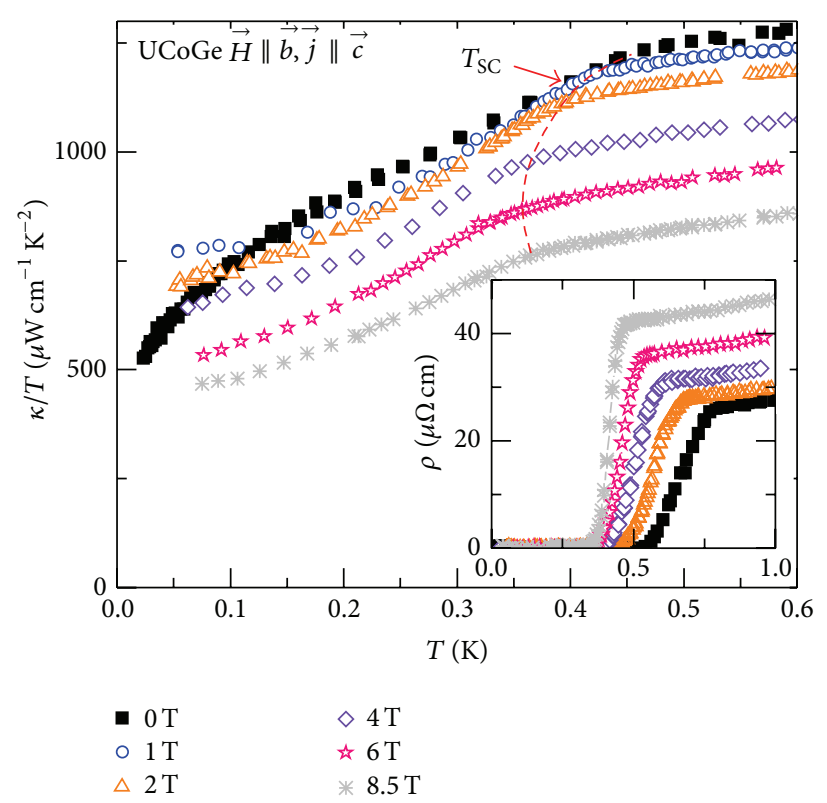

(a)

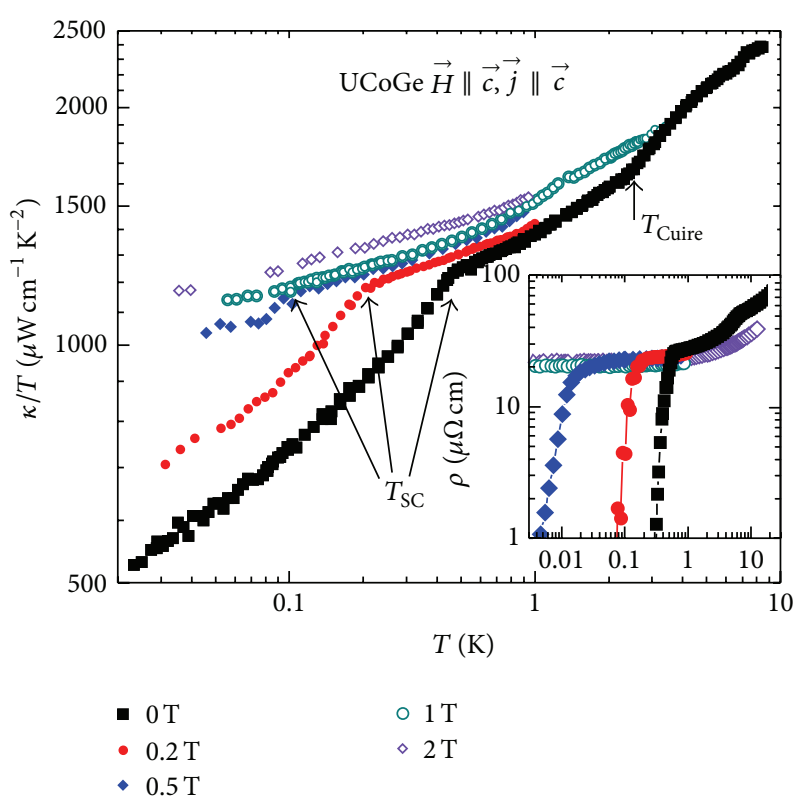

(b)

Figure 1: Raw data for thermal conductivity divided by temperature and electrical resistivity in inset, with (a) $\vec{H} \| \vec{b}$-axis and (b) $\vec{H} \| \vec{c}$-axis. Heat current is applied along the $\vec{c}$ crystallographic axis.

with a line of nodes in the parent system URhGe. However, in contradiction to the theoretical prediction this line was proposed to occur at $k_{x}=0$. Thermal conductivity is a strong probe of the gap symmetry of a superconductor. Indeed, the density of nonsuperconducting quasiparticles, which are the main heat carrier channel at low temperatures, is strongly influenced by the presence and type of gap nodes.

\section{Method and Raw Data}

We measured thermal conductivity $(\kappa)$ with a twothermometer one-heater setup in the temperature range $30 \mathrm{mK}-10 \mathrm{~K}$ and in magnetic field up to 8.5 Tesla. Resistive carbon thermometers were used, held by thin Kevlar strings, and measured through superconducting $\mathrm{NbTi}$ wires to insure good external thermal insulation. Thermometers and sample were connected through a gold wire spot welded on the sample side, achieving a contact resistance of $\sim 15 \mu \Omega$. Fourprobe electrical resistivity $(\rho)$ was measured simultaneously using the same gold wires for voltage measurement, allowing a direct verification of the setup using the Wiedemann-Franz law $\left(\kappa \rho / T \rightarrow L_{0}\right.$ for $T \rightarrow 0$ with $\left.L_{0}=2.44 \cdot 10^{-8} \mathrm{~W} \Omega \mathrm{K}^{-2}\right)$. This setup was mounted on a piezo rotator which allows fine tuning of the relative angle between magnetic field and sample crystallographic axis. This is required due to the strong angular dependence of magnetic properties [6]. The single crystal of pure UCoGe composition was grown using the Czochralski method in a tetra-arc furnace, characterized by Laue diffraction and specific heat. The sample was cut in a bar shape with the widest direction of $l \simeq 2 \mathrm{~mm}$ along the $\vec{c}$ crystallographic axes. With a residual resistivity ratio of $R R R \cong 16$, the sample offers a good compromise between crystallographic quality (single grain crystal) and low mean free path. The latter property is required in order to differentiate between electronic and magnetic thermal conductivity contributions as described below.

Thermal conductivity divided by temperature $(\kappa / T)$ and resistivity $(\rho)$ for two field orientations, $\vec{H} \| \vec{b}$-axis and $\vec{H} \| \vec{c}$ axis, are presented in Figures 1(a) and 1(b) respectively. The ferromagnetic transition ( $T_{\text {Curie }}$ ) disappears with a magnetic field applied along the $\vec{c}$ crystallographic axis in agreement with such an orientation for the magnetic moments, due to the absence of symmetry breaking at the transition. The superconducting transition $\left(T_{\mathrm{SC}}\right)$ is clearly observed by a kink in the two sets of curves below $\sim 0.5 \mathrm{~K}$. The observation of both superconducting and ferromagnetic transitions in thermal conductivity curves indicates that both phases are bulk. The enhancement of $T_{\mathrm{SC}}$ under magnetic field $(\vec{H} \| \vec{b})$ previously observed in resistivity measurements [6] is confirmed as a bulk property (red dashed line in Figure 1(a)) which indicates a high precision in the sample alignment. Indeed, such enhancement was reported to occur only when the magnetic field is applied within $1^{\circ}$ of the $\vec{b}$ crystallographic axis [17]. It is particularly interesting to note that the bulk reentrance of superconductivity already occurs at $\sim 5$ Tesla, while the resistive one is only observed around 10 Tesla [6]. The method used to extract $T_{\mathrm{SC}}$ is described in [18]. The residual value in the superconducting state is quite large ( $50 \%$ of the normal state value). For a fully open superconducting gap (s-wave) the ratio $\kappa / T$ vanishes with temperature $\left(\lim _{T \rightarrow 0} \kappa / T=0\right.$ ), as observed in $\mathrm{Nb}$ [19], NbSe [20], and $\mathrm{MgB}_{2}$ [21], for example. For systems in 
which the superconducting gap vanishes at points or lines of nodes, a residual value is expected due to impurity scattering. Such residual value was observed in the d-wave high- $T_{\mathrm{SC}}$ superconductor $\mathrm{Tl}_{2} \mathrm{Ba}_{2} \mathrm{CuO}_{6+\delta}$ [22] and in the possibly pwave superconductor $\mathrm{Sr}_{2} \mathrm{RuO}_{4}[23,24]$. For systems in which a superconducting gap with lines of nodes is present, the limit can even be universal, with $\left(\kappa(T) T_{\mathrm{SC}} /\left(\kappa\left(T_{\mathrm{SC}}\right) T\right)\right)_{T \rightarrow 0}=C$ independent of the amount of impurities [25].

In $\mathrm{UCoGe}$, the residual value of thermal conductivity is not universal. A recently probed sample with $\mathrm{RRR} \cong$ 100 has a lower residual term of $\sim 30 \%$ of the extrapolated nonsuperconducting value [26]. The residual term can be due to the presence of nodes in the superconducting gap, a special superconducting phase (like superfluid phase Al of ${ }^{3} \mathrm{He}$ ), a band of gapless superconductivity (due to impurities), or an inhomogeneous sample (partly nonsuperconducting). This last option is supported by NQR measurements, reporting a mixture of superconducting and nonsuperconducting regions [4], and by a specific heat measurement reporting a residual term of about $50 \%$ of the normal state value [27]. Both experiments were performed on samples of similar quality to the one used in this study. Note that the sharpness of both the ferromagnetic and the superconducting transitions exclude the possibility of a distribution of transitions in the bulk phases, which would give rise to broad or no features in thermal conductivity. The higher value for $T_{\mathrm{SC}}$ observed in the resistivity measurement must originate from part of the sample with nonbulk superconductivity. This could be due to filamentary superconductivity or superconductivity occurring first in ferromagnetic domain wall, where magnetization is suppressed which is certainly favorable for superconductivity.

\section{Results and Discussion}

In Figure 2 the Lorenz ratio $\left(L / L_{0}=\kappa \rho /\left(T L_{0}\right)\right)$ calculated for different values of the magnetic field applied along the $\vec{b}$ crystallographic direction is plotted. For $T \rightarrow 0$ the Wiedemann-Franz law $L=L_{0}$ is obeyed within $5 \%$ which reflect the validity of our measurements. The equality indicates that, at low temperatures, electrical and thermal currents are transported by the same carriers: the electrons. At finite temperatures two effects can produce a deviation from the unity of $L / L_{0}$. Other thermal carrier channels such as phonon or magnon excitations will enhance this ratio. In contrary the ratio will be lower than one if a strong electron-electron inelastic scattering, reducing the efficiency of electronic thermal conductivity, dominates [28]. In a conventional metal, magnons are absent and as few phonons are present at low temperatures, the ratio is reduced below 1 for the lowest temperatures and exceeds 1 in the higher temperatures regime due to the phonons contribution. The depth of the minimum in $L / L_{0}$ depends on the mean free path of the quasiparticles.

As the sample investigated has a relatively low RRR value the mean free path of the quasiparticles is short and only a weak deviation from 1 is expected for $L / L_{0}$ at low temperatures. In Figure 2 the increase of $L / L_{0}$ above 1 from

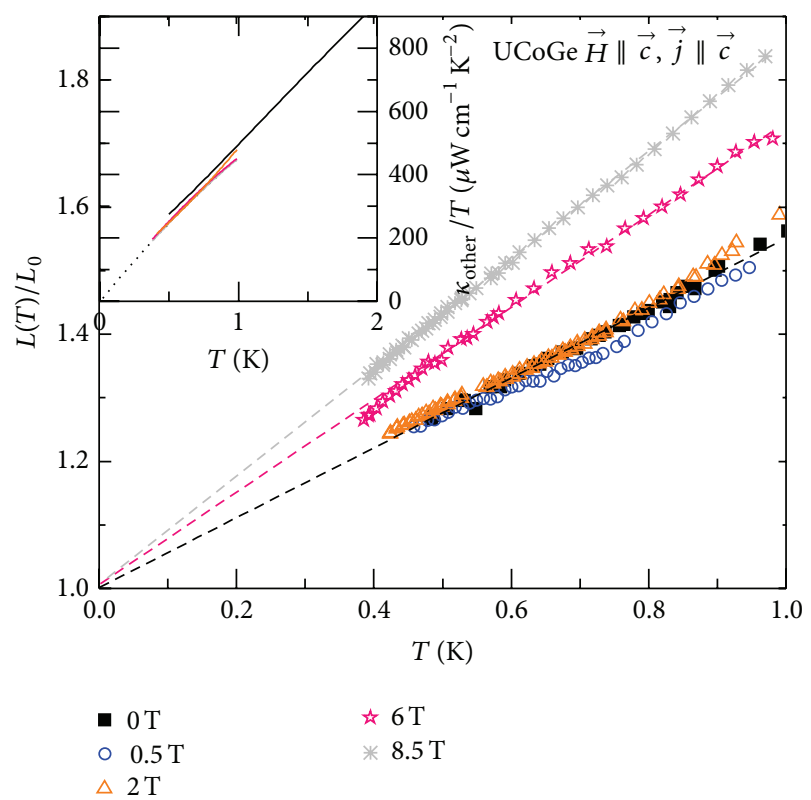

FIGURE 2: Lorenz ratio $\kappa \rho /\left(L_{0} T\right)$ obtained from the thermal conductivity and resistivity data presented in Figure 1(a). All the curves extrapolate to 1 at $T=0 \mathrm{~K}$, reflecting the good quality of the measurement. In inset the residual thermal conductivity contribution $\left(\kappa_{\text {other }} / T\right)$ is shown (see text).

the lowest temperatures indicates the presence of another type of heat carrier than the electrons. As UCoGe is ferromagnetic, magnons or uniaxial fluctuations are good candidates $[5,18,29]$. An analysis of the anisotropy of this contribution with heat current direction reenforce this hypothesis [26]. We can obtain the approximate value of this additional thermal conductivity contribution $\left(\kappa_{\text {other }}\right)$ by assuming the Lorenz ratio is 1 for the electronic contribution: $L=\left(\kappa_{\mathrm{el}}+\kappa_{\mathrm{other}}\right) \rho / T$ with $L_{0}=\kappa_{\mathrm{el}} \rho / T \rightarrow \kappa_{\text {other }} / T=\left(L-L_{0}\right) / \rho$. We found that the magnetic contribution is independent of a magnetic field applied along the $\vec{b}$ crystallographic direction (inset of Figure 2) while it is strongly reduced by a magnetic field applied in the $\vec{c}$ crystallographic direction, as expected for longitudinal spin fluctuations [18] and in agreement with a previous study using a different technique to extract the magnetic contribution [26].

In order to further analyze the temperature dependence of thermal conductivity, we subtract the additional contribution obtained previously $\left(\kappa_{S}=\kappa-\kappa_{\text {other }}\right)$ and calculate the thermal conductivity one would observe if the compound was not superconducting, hereafter called normal contribution to thermal conductivity $\left(\kappa_{N}\right)$. This is done by extrapolating the normal state resistivity to $T \rightarrow 0$ assuming a Fermi liquid dependence $\left(\rho(T)=\rho_{0}+A T^{2}\right)$. Then we calculated the electronic contribution to thermal conductivity using the Wiedemann-Franz law: $\kappa_{N} / T=L_{0} / \rho$. Note that this is only possible due to the moderate RRR value of the sample, when practically no deviation from the WiedemannFranz law is expected. Indeed, for a more general study [26], a phenomenological model had to be introduced for the electronic thermal conductivity which prevents any further discussion of the temperature dependence. 

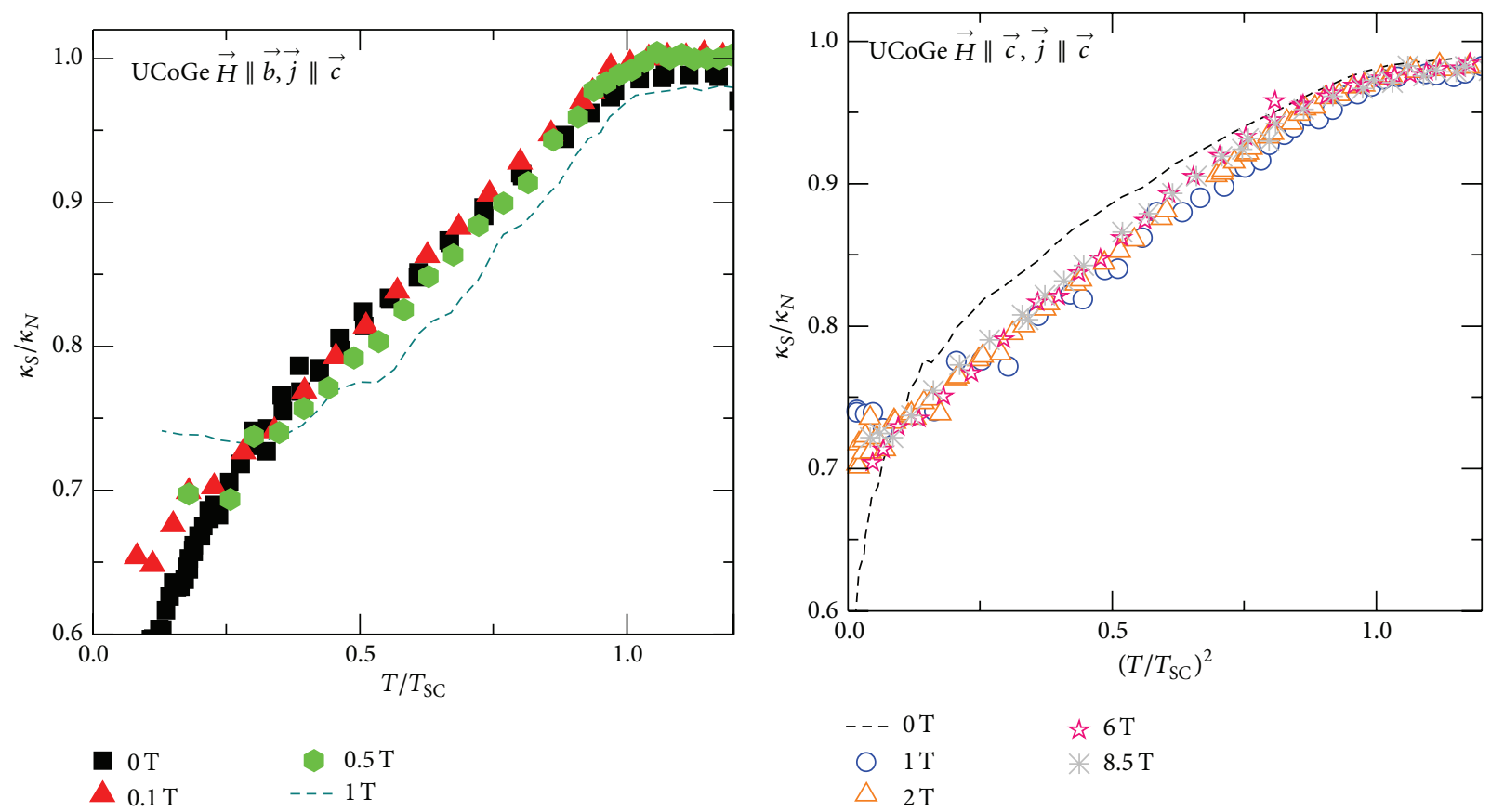

(a)

(b)

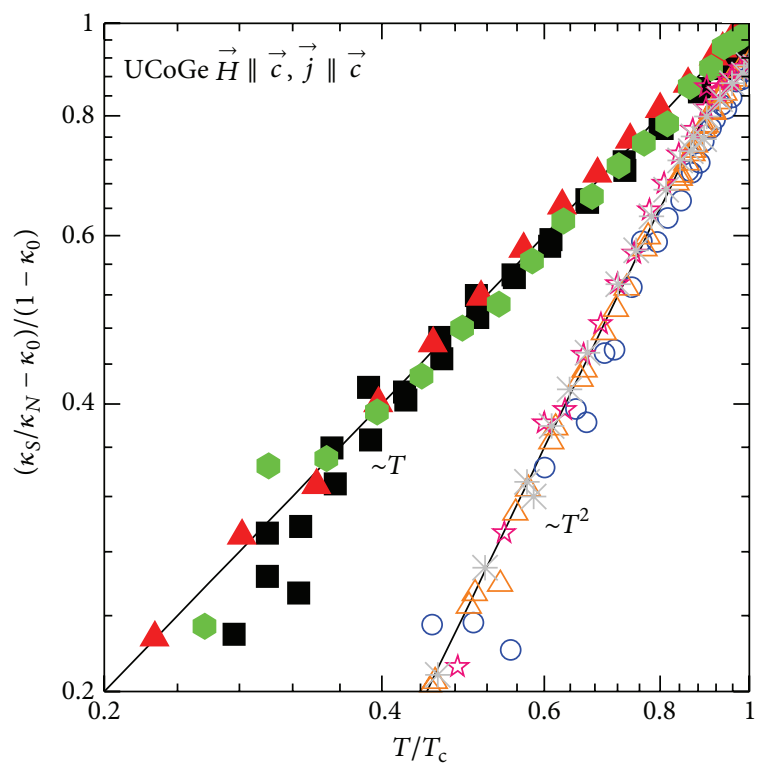

$\begin{array}{ll}\square 0 \mathrm{~T} & \triangle 2 \mathrm{~T} \\ 0.1 \mathrm{~T} & \text { 尔 } 6 \mathrm{~T} \\ 0.5 \mathrm{~T} & * 8.5 \mathrm{~T} \\ 01 \mathrm{~T} & \end{array}$

(c)

FIGURE 3: Ratio of the superconducting to normal state value of the electronic contribution to thermal conductivity. (a) This ratio is linear in temperature at low fields $\mu_{0} H<1 \mathrm{~T}$ and quadratic in temperature for higher fields (b). (c) The two temperature dependencies are best viewed in a double logarithmic plot after subtraction of the residual term $\kappa_{0} . \kappa_{0}=\lim _{T \rightarrow 0} \kappa_{S} / \kappa_{N}(T)$ was linearly extrapolated from panels (a) and (b) in the temperature ranges $0.25<T / T_{c}<0.97$ and $0.08<\left(T / T_{c}\right)^{2}<0.97$, respectively. 


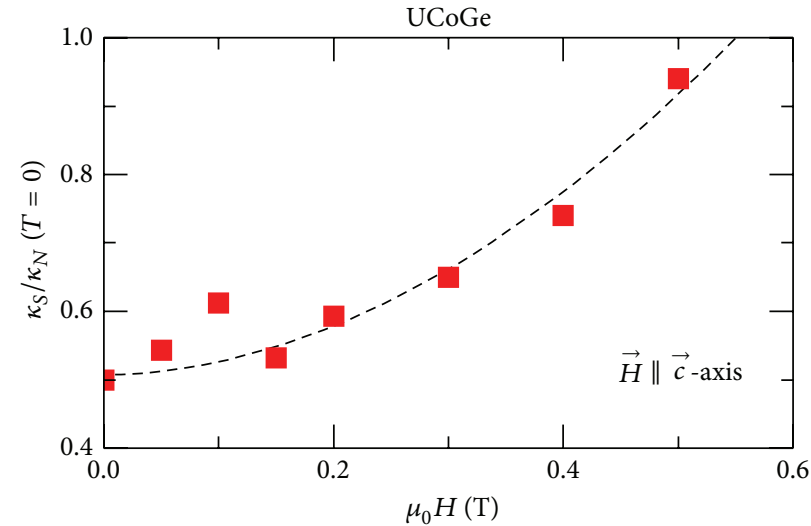

(a)

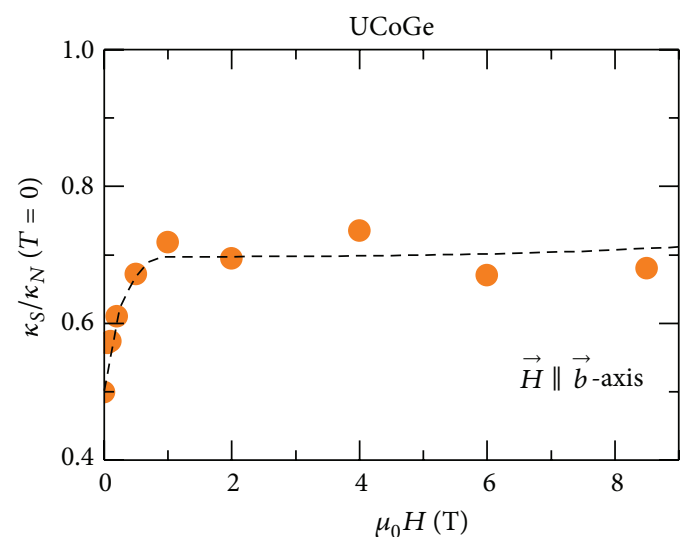

(b)

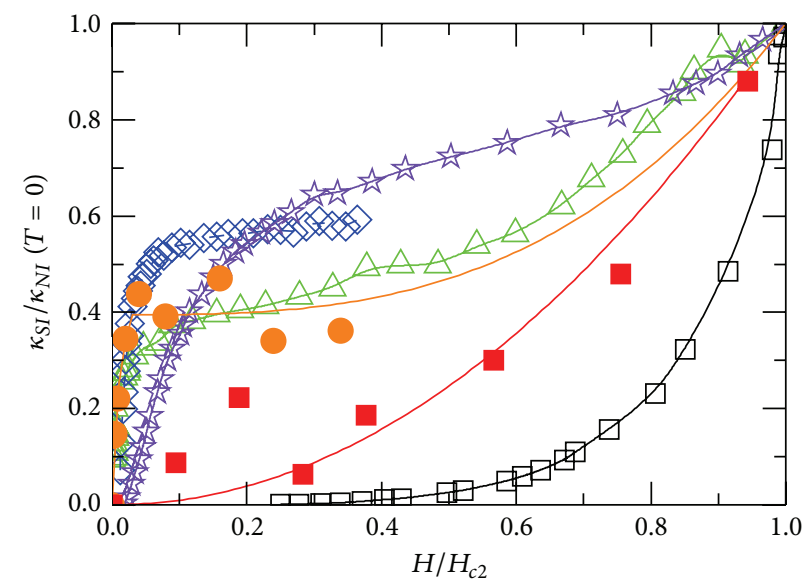

(c)

Figure 4: Field dependence of the ratio $\kappa_{S}(H) / \kappa_{N}(H)$ : (a) $\vec{H} \| \vec{c}$ (red squares) and (b) $\vec{H} \| \vec{b}$ (orange circles). (c) Comparison between $\kappa_{S 1}(H) / \kappa_{N 1}(H)$ (see text) of UCoGe and the single gap superconductor $\mathrm{Nb}$ (black squares [19]) and the two-band superconductors $\mathrm{PrOs}_{4} \mathrm{Sb}_{12}$ (green triangles [10]), $\mathrm{MgB}_{2} \vec{H} \|(a, b)$ (blue diamonds [21]), and $\mathrm{MgB}_{2} \vec{H} \| \vec{c}$ (violet stars [21]). $H_{c 2} \| \vec{b}$ of UCoGe is taken as 25 Tesla in (c).

The ratio of superconducting to "extrapolated normal state" thermal conductivity in the superconducting state $\left(\kappa_{S} / \kappa_{N}\right)$ is reported in Figures 3(a) and 3(b) for different magnetic fields applied along the $\vec{b}$ crystallographic direction. Such ratio is related to the fraction of superfluid quasiparticles. We can clearly distinguish two different temperatures dependencies: linear for $\mu_{0} H<1 \mathrm{~T}$ and quadratic otherwise. Figure 3(c) emphasizes the linear and quadratic temperature dependencies with a double logarithmic plot. The temperature dependence of thermal conductivity is related to the type of nodes (points and lines) and their opening angle [30]. The different temperature dependencies indicate two different gap structures depending on the field range. There is no report of a phase transition between two different superconducting phases, neither with temperature nor upon applying a magnetic field, as required in order to modify the symmetry of the superconducting gap and our measurements support a crossover. Therefore, we infer a multigap superconducting state and not multiple superconducting states. The low field temperature dependence would then result from the addition of the thermal conductivity of the two bands, while at high fields only the band with the larger superconducting gap would be superconducting. The high magnetic field temperature dependence, $\kappa_{S} / \kappa_{N} \sim$ $T^{2}$, suggests the presence of a line of nodes in the gap of the superconducting band, as expected in analogy to URhGe [16]. Note that the observation of two different field ranges is independent of the temperature dependence of the subtracted $\kappa_{\text {other }}$ contribution. A similar evolution of the temperature dependence of thermal conductivity was observed in well-known two-band superconductors such as $\mathrm{MgB}_{2}$ [21], CeCoIn 5 [11], and $\mathrm{NbSe}_{2}$ [20] although with different power laws. The small deviation from linearity of $\kappa_{S} / \kappa_{N}$ at low temperatures $\left(T / T_{\mathrm{SC}}<0.25\right)$ for $\mu_{0} H=0 \mathrm{~T}$ is understood in the multigap scenario as corresponding to the characteristic energy of the smaller gap (Figures 1(a) and 3).

The idea of a multigap superconductivity is reenforced by the appearance of a plateau above $\mu_{0} H>1$ T in the field evolution of the residual term of thermal conductivity, for $\vec{H} \| \vec{b}$ (Figure 4(b)). The experimental resolution might not allow observing such feature for $\vec{H} \| \vec{c}$ (Figure 4(a)). The decoupling 
between the two gaps might also be weaker in this configuration. If we assume that the residual term for $\mu_{0} H=$ $0 \mathrm{~T}$ is due to an inhomogeneous part of the sample never superconducting, we can extend the analysis by comparing UCoGe to well established two gaps superconductors, as $\mathrm{PrOs}_{4} \mathrm{Sb}_{12}$ and $\mathrm{MgB}_{2}$ (Figure 4(c)). Here we have assumed two parallel contributions to thermal conductivity $\kappa_{i}=\kappa_{i 1}+$ $\kappa_{2}$, with $(i=S, N)$ and $\kappa_{2}$ for the never superconducting contribution. $\kappa_{2}$ is assumed to be field independent. Independently of this assumption, the three systems, UCoGe, $\mathrm{PrOs}_{4} \mathrm{Sb}_{12}$, and $\mathrm{MgB}_{2}$, presented in Figure 4 are characterized by two energy scales corresponding to the values of the two respective gaps. The field dependence of $\kappa_{S} / \kappa_{N}$ for a single gap superconductor is drastically different to the one found in UCoGe as demonstrated with the case of $\mathrm{Nb}$.

A ferromagnetic system has inherently two bands, for majority and minority electron spins. It is therefore tempting to map the two superconducting gaps to the two ferromagnetic bands. The different strength of superconductivity could then be explained by the proximity to the Lifshitz phase transition, previously reported [31]. The strong increase of density of state would enhance the superconducting coupling. It is however not clear whether the two electronic bands would be decoupled sufficiently enough to induce multigap superconductivity. A classical scenario of the two gaps occurring on different Fermi pockets is another possibility.

\section{Conclusions}

In conclusion, with the study of thermal conductivity $(\kappa(T, H))$ in the ferromagnetic heavy fermion system UCoGe, we confirm the reenforcement of superconductivity under magnetic field and establish the bulk character of this effect. In addition, we observed two distinct energy scales depending on the value of the magnetic field applied along the $\vec{b}$ crystallographic axis. At high magnetic fields the temperature dependence of thermal conductivity is compatible with the presence of a line of nodes in the superconducting gap as reported in the parent system URhGe. Both the field dependence of the residual term and the different temperature dependencies of $\kappa(T, H)$ suggest the realization of multigap superconductivity. Further experiments on samples with different RRR values and heat currents directions as well as theoretical modeling of thermal conductivity in multigap systems are required in order to obtain the exact nature of the superconducting state in the ferromagnetic superconductor UCoGe.

\section{Conflict of Interests}

The authors declare that there is no conflict of interests regarding the publication of this paper.

\section{Acknowledgments}

The authors gratefully acknowledged experimental support from J. P. Brison and sample preparation from V. Taufour.
This work was supported by the Commissariat à l'énergie atomique of Grenoble and by the Université de Grenoble.

\section{References}

[1] N. T. Huy, A. Gasparini, D. E. de Nijs et al., "Superconductivity on the border of weak itinerant ferromagnetism in UCoGe," Physical Review Letters, vol. 99, no. 6, Article ID 067006, 4 pages, 2007.

[2] N. T. Huy, D. E. de Nijs, Y. K. Huang, and A. de Visser, "Unusual upper critical field of the ferromagnetic superconductor UCoGe," Physical Review Letters, vol. 100, no. 7, Article ID 077002, 2008.

[3] A. de Visser, N. T. Huy, A. Gasparini et al., "Muon spin rotation and relaxation in the superconducting ferromagnet UCoGe," Physical Review Letters, vol. 102, no. 16, Article ID 167003, 2009.

[4] T. Ohta, T. Hattori, K. Ishida et al., "Microscopic coexistence of ferromagnetism and superconductivity in single-crystal UCoGe," Journal of the Physical Society of Japan, vol. 79, no. 2, Article ID 023707, 5 pages, 2010.

[5] T. Ohta, Y. Nakai, Y. Ihara et al., "Ferromagnetic quantum critical fluctuations and anomalous coexistence of ferromagnetism and superconductivity in UCoGe revealed by Co-NMR and NQR studies," Journal of the Physical Society of Japan, vol. 77, Article ID 023707, 5 pages, 2008.

[6] D. Aoki, T. D. Matsuda, V. Taufour, E. Hassinger, G. Knebel, and J. Flouquet, "Extremely large and anisotropic upper critical field and the ferromagnetic instability in UCoGe," Journal of the Physical Society of Japan, vol. 78, no. 11, Article ID 113709, 4 pages, 2009.

[7] V. P. Mineev, "Paramagnetic limit in ferromagnetic superconductors with triplet pairing," Physical Review B, vol. 81, Article ID 180504, 4 pages, 2010.

[8] G. Binnig, A. Baratoff, H. E. Hoenig, and J. G. Bednorz, "Twoband superconductivity in $\mathrm{Nb}$-doped $\mathrm{SrTiO}_{3}$," Physical Review Letters, vol. 45, no. 16, pp. 1352-1355, 1980.

[9] F. Bouquet, Y. Wang, I. Sheikin et al., "Specific heat of single crystal $\mathrm{MgB}_{2}$ : a two-band superconductor with two different anisotropies," Physical Review Letters, vol. 89, Article ID 257001, 2002.

[10] G. Seyfarth, J. P. Brison, M.-A. Méasson et al., "Multiband superconductivity in the heavy fermion compound $\mathrm{PrOs}_{4} \mathrm{Sb}_{12}$," Physical Review Letters, vol. 95, no. 10, Article ID 107004, 2005.

[11] G. Seyfarth, J. P. Brison, G. Knebel, D. Aoki, G. Lapertot, and J. Flouquet, "Multigap superconductivity in the heavy-fermion system CeCoIn 5 ," Physical Review Letters, vol. 101, no. 4, Article ID 046401, 2008.

[12] R. Khasanov, A. Shengelaya, A. Maisuradze et al., "Experimental evidence for two gaps in the high-temperature $\mathrm{La}_{1.83} \mathrm{Sr}_{0.17} \mathrm{CuO}_{4}$ superconductor," Physical Review Letters, vol. 98, no. 5, Article ID 057007, 2007.

[13] J. Yang and G. Q. Zheng, "Multiple superconducting gaps, anisotropic spin fluctuations and spin-orbit coupling in ironpnictides," Modern Physics Letters B, vol. 26, no. 15, Article ID 1230008, 20 pages, 2012.

[14] M. Samsel-Czekała, S. Elgazzar, P. M. Oppeneer, E. Talik, W. Walerczyk, and R. Troć, "The electronic structure of UCoGe by ab initio calculations and XPS experiment," Journal of Physics Condensed Matter, vol. 22, no. 1, Article ID 015503, 2010.

[15] V. P. Mineev and T. Champel, "Theory of superconductivity in ferromagnetic superconductors with triplet pairing," Physical 
Review B: Condensed Matter and Materials Physics, vol. 69, no. 14, Article ID 144521, 2004.

[16] F. Hardy and A. D. Huxley, "P-Wave superconductivity in the ferromagnetic superconductor URhGe," Physical Review Letters, vol. 94, no. 24, Article ID 247006, 2005.

[17] D. Aoki, T. D. Matsuda, F. Hardy et al., "Superconductivity reinforced by magnetic field and the magnetic instability in uranium ferromagnets," Journal of the Physical Society of Japan, vol. 80, supplement A, Article ID SA008, 6 pages, 2011.

[18] L. Howald, Interactions between superconductivity and quantum criticality in CeCoIn, URhGe and UCoGe [Ph.D. thesis], Université de Grenoble, 2011, http://tel.archives-ouvertes.fr/tel00584598.

[19] J. Lowell and J. B. Sousa, "Mixed-state thermal conductivity of type II superconductors," Journal of Low Temperature Physics, vol. 3, no. 1, pp. 65-87, 1970.

[20] E. Boaknin, M. A. Tanatar, J. Paglione et al., "Heat conduction in the vortex state of $\mathrm{NbSe}_{2}$ : evidence for multiband superconductivity," Physical Review Letters, vol. 90, no. 11, Article ID 117003, 2003.

[21] A. V. Sologubenko, J. Jun, S. M. Kazakov, J. Karpinski, and H. R. Ott, "Thermal conductivity of single-crystalline $\mathrm{MgB}_{2}$," Physical Review B: Condensed Matter and Materials Physics, vol. 66, no. 1, Article ID 014504, 2002.

[22] C. Proust, E. Boaknin, R. W. Hill, L. Taillefer, and A. P. Mackenzie, "Heat transport in a strongly overdoped cuprate: Fermi liquid and a pure d-wave BCS superconductor," Physical Review Letters, vol. 89, no. 14, Article ID 147003, 2002.

[23] H. Suderow, J. P. Brison, J. Flouquet, A. W. Tyler, and Y. Maeno, "Very low temperature thermal conductivity in the layered perovskite superconductor $\mathrm{Sr}_{2} \mathrm{RuO}_{4}$," Journal of Physics Condensed Matter, vol. 10, no. 34, pp. L597-L602, 1998.

[24] M. Suzuki, M. A. Tanatar, N. Kikugawa, Z. O. Mao, Y. Maeno, and T. Ishiguro, "Universal heat transport in $\mathrm{Sr}_{2} \mathrm{RuO}_{4}$," Physical Review Letters, vol. 88, Article ID 227004, 2002.

[25] M. J. Graf, S.-K. Yip, J. A. Sauls, and D. Rainer, "Electronic thermal conductivity and the Wiedemann-Franz law for unconventional superconductors," Physical Review B-Condensed Matter and Materials Physics, vol. 53, no. 22, pp. 15147-15161, 1996.

[26] M. Taupin, L. Howald, D. Aoki, J. Flouquet, and J. P. Brison, "Existence of anisotropic spin fluctuations at low temperature in the normal phase of the superconducting ferromagnet UCoGe," Physical Review B, vol. 89, Article ID 041108, 2014.

[27] D. Aoki, M. Taupin, C. Paulsen et al., "Field-induced phenomena in ferromagnetic superconductors UCoGe and URhGe," Journal of the Physical Society of Japan, vol. 81, Article ID SB002, 6 pages, 2012.

[28] J. M. Ziman, Principles of the Theory of Solids, Cambridge University Press, London, UK, 2nd edition, 1979.

[29] K. Prokeš, A. de Visser, Y. K. Huang, B. Fåk, and E. Ressouche, "Anomalous spin distribution in the superconducting ferromagnet UCoGe studied by polarized neutron diffraction," Physical Review B, vol. 81, Article ID 180407, 2010.

[30] Y. Matsuda, K. Izawa, and I. Vekhter, "Nodal structure of unconventional superconductors probed by angle resolved thermal transport measurements," Journal of Physics: Condensed Matter, vol. 18, no. 44, pp. R705-R752, 2006.

[31] L. Malone, L. Howald, A. Pourret et al., "Thermoelectricity of the ferromagnetic superconductor UCoGe," Physical Review B-Condensed Matter and Materials Physics, vol. 85, no. 2, Article ID 024526, 2012. 

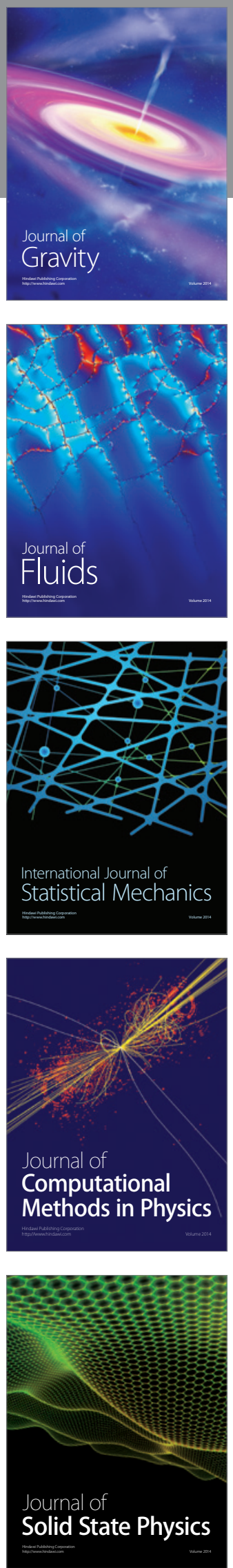

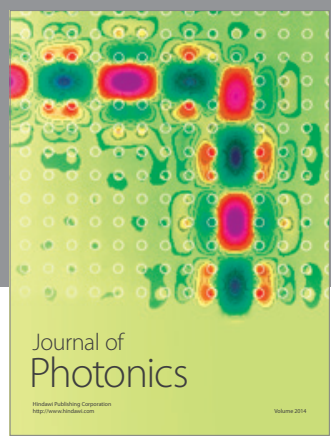

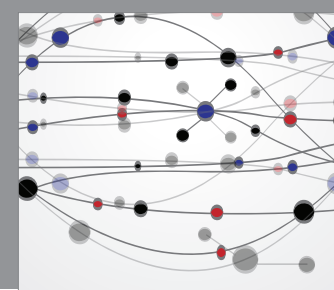

The Scientific World Journal

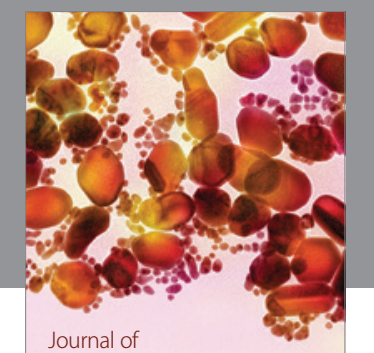

Soft Matter
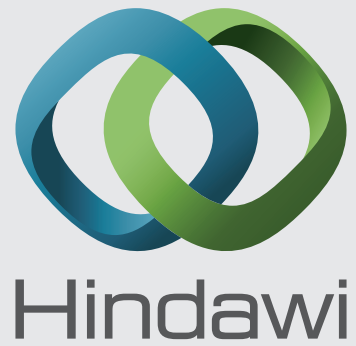

Submit your manuscripts at

http://www.hindawi.com
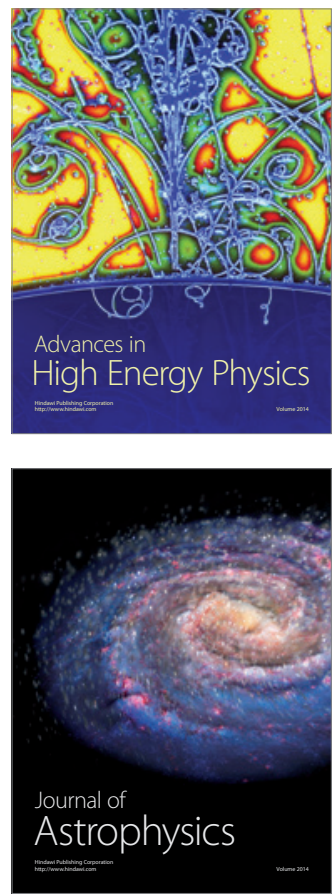
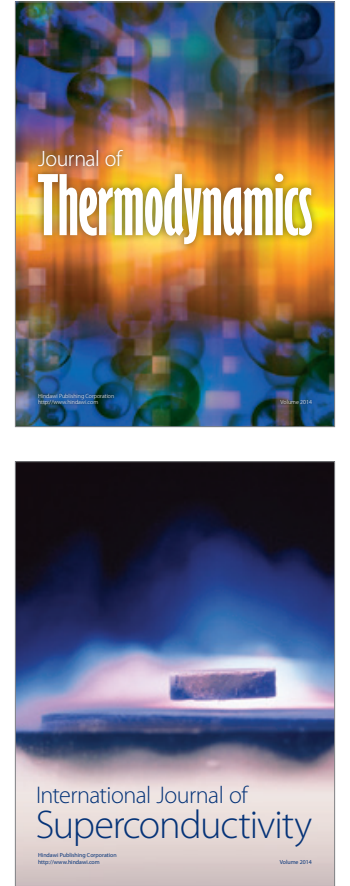
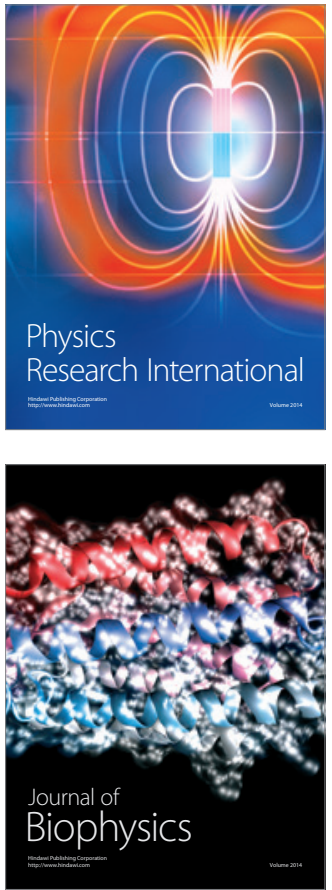
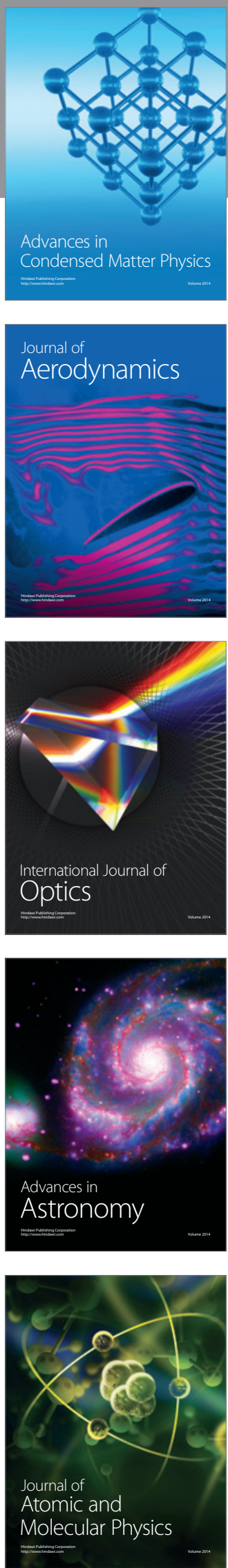